\title{
The What, When, Who, Why, How and Where of Myocardial Infarction With Non-Obstructive Coronary Arteries (MINOCA)
}

\author{
Sivabaskari Pasupathy, BSc; Rosanna Tavella, BSc, PhD; John F. Beltrame, BSc, MD, PhD
}

\begin{abstract}
Myocardial infarction with non-obstructive coronary arteries (MINOCA) is an intriguing clinical entity that is being increasingly recognized with the more common use of coronary angiography during acute myocardial infarction. This review systematically addresses the contemporary understanding of MINOCA, including, (1) what are the diagnostic criteria, (2) when the diagnosis should be considered, (3) who is at risk, (4) why this new syndrome should be diagnosed, (5) how these patients should be managed, and (6) where to next? (Circ $J$ 2016; 80: 11-16)
\end{abstract}

Key Words: Coronary angiography; Coronary artery disease; Coronary spasm; MINOCA; Myocardial infarction

$\mathbf{O}$ ur understanding of acute myocardial infarction (AMI) has evolved considerably over the past 50 years, which has given rise to innovative therapies that have improved patient outcomes. These innovations have primarily focused on alleviating atherothrombotic processes that obstruct coronary blood flow, evident in most patients with AMI. However, in approximately 1 in 10 patients (5-14\% reported prevalence $^{1-4}$ ) with AMI, angiography does not reveal obstructive coronary artery disease (CAD), so the pathophysiological processes responsible for the AMI are not immediately evident. These patients are labeled as having "myocardial infarction with non-obstructive coronary arteries (MINOCA)", which is being increasingly recognized with the more widespread use of coronary angiography in AMI. Importantly, the management of these intriguing patients is predicated upon their initial recognition and subsequent evaluation to elucidate the pathophysiological processes responsible for their presentation.

Since the term was first coined, ${ }^{5}$ clinical interest in patients with MINOCA has rapidly evolved and focused attention on the condition. The purpose of this review is to summarize the contemporary understanding of MINOCA by adopting the Kipling approach (known as the 'Five Ws and One H') as detailed in his classic novel, the 'Just so stories': 'I keep six honest serving men (they taught me all I knew); their names are what and why and when and how and where and who.' ${ }^{6}$

\section{WHAT IS MINOCA?}

The diagnosis of MINOCA is made in a patient presenting with diagnostic features of an AMI, in whom angiography (either invasive or CT angiography) does not show obstructive $\mathrm{CAD}$, and there is no immediately apparent cause for the presentation. Specifically, the diagnostic criteria include the fol- lowing 3 conditions.

1. AMI criteria, including:

a. Positive cardiac biomarker: defined as a rise and/or fall in serial levels, with at least 1 value above the 99th percentile upper reference limit.

b. Clinical evidence of MI, including any of the following:

i. Ischemic symptoms (chest pain and/or dyspnea)

ii. Ischemic ECG changes (new ST segment changes or left bundle branch block)

iii. New pathological $Q$ waves

iv. New loss of viable myocardium on myocardial perfusion imaging or

v. New regional wall motion abnormality on left ventricular imaging.

2. Absence of obstructive $\mathrm{CAD}$ on angiography (defined as no lesions $\geq 50 \%$ ).

3. No clinically apparent cause for the acute presentation.

These diagnostic criteria incorporate contemporary definitions of $\mathrm{AMI}^{7}$ and non-obstructive $\mathrm{CAD},{ }^{8}$ whereas some earlier studies have used less conventional criteria. Although patients may have fulfilled the established criteria for AMI, some will have experienced disorders that 'mimic AMI' and thus must be distinguished from those with an 'ischemic AMI' (as outlined later).

The diagnosis of MINOCA should be considered as a "working diagnosis". Thus, just as a diagnosis of "heart failure" necessitates an evaluation of the underlying cause for the heart failure presentation, a diagnosis of MINOCA should prompt investigation for its underlying cause.

Figure 1 summarizes these principles, detailing the features of a 55-year-old woman with (1) an acute anterior ST-

Received October 13, 2015; revised manuscript received October 26, 2015; accepted October 27, 2015; released online November 20,2015

Discipline of Medicine, University of Adelaide, Adelaide, South Australia; Central Adelaide Local Health Network, SA Health, Adelaide, South Australia, Australia

Mailing address: John F. Beltrame, Professor, BSc, MD, PhD, Discipline of Medicine, The Queen Elizabeth Hospital / University of Adelaide, 28 Woodville Road, Woodville South, Adelaide, SA 5011, Australia. E-mail: john.beltrame@adelaide.edu.au

ISSN-1346-9843 doi:10.1253/circj.CJ-15-1096

All rights are reserved to the Japanese Circulation Society. For permissions, please e-mail: cj@j-circ.or.jp 


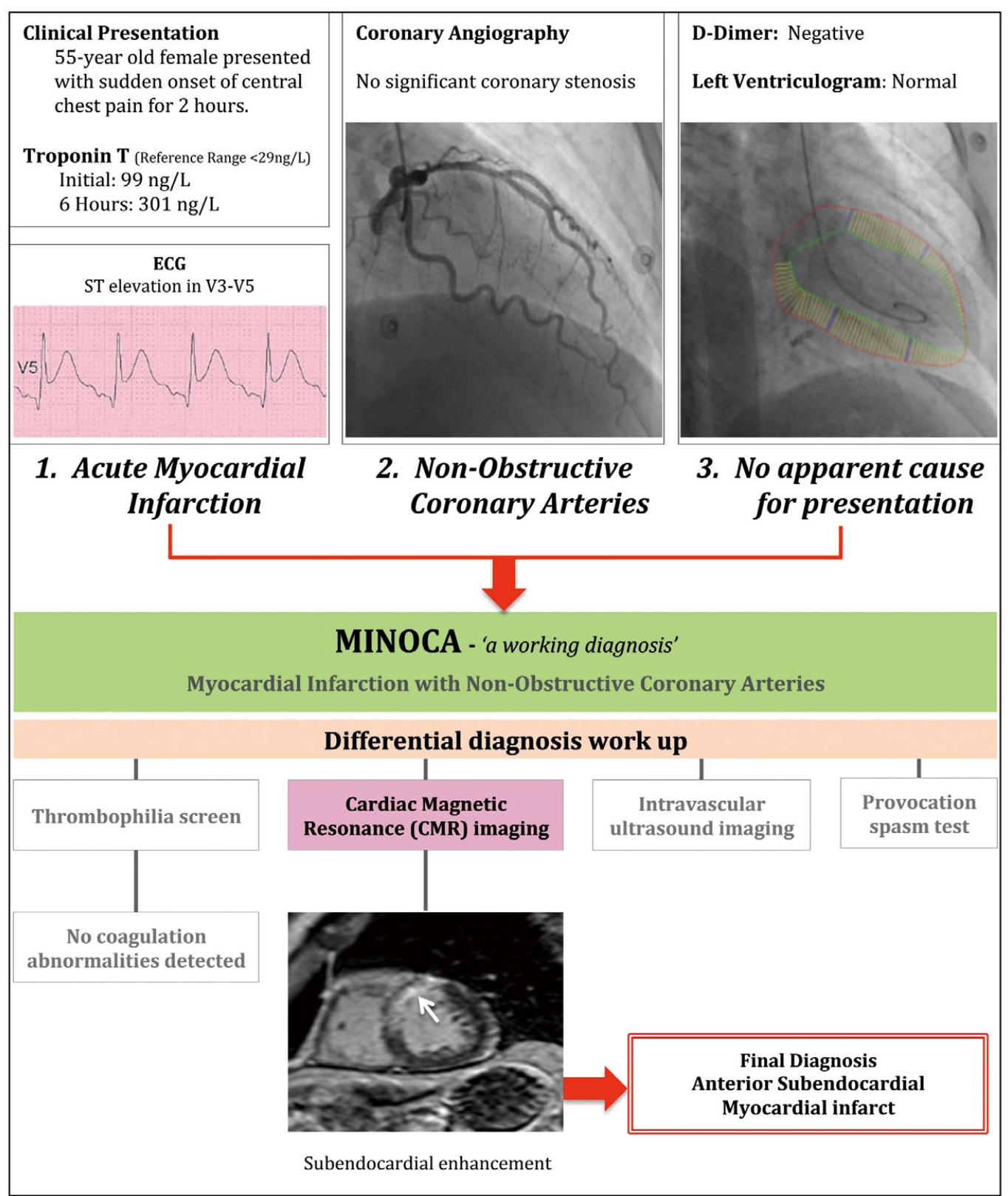

Figure 1. Myocardial infarction with non-obstructive coronary arteries (MINOCA): a case study of a 55-year-old woman with an acute anterior ST-elevation MI presentation. See text for descriptive details.

elevation MI presentation, subsequently shown to have (2) non-obstructive coronary arteries on urgent angiography, and (3) no apparent cause for the AMI presentation at angiography because left ventriculography shows no evidence of takotsubo or other cardiomyopathies, and the D-dimer is negative. Accordingly, a working diagnosis of MINOCA was made and a search for the underlying cause undertaken. Cardiac magnetic resonance (CMR) imaging revealed an anterior subendocardial myocardial infarct and the patient was discharged with aspirin, statin and a calcium-channel blocker on the presumption that the infarct arose from either plaque disruption or coronary spasm. In some institutions, further delineation of the underlying cause may be considered, with such investigations as intravascular ultrasound imaging, provocative spasm testing and thrombophilic disorder screening (Table).

\section{WHEN to Diagnose MINOCA?}

The diagnosis of MINOCA should be made upon completion of coronary angiography in patients presenting with an AMI. As discussed, the diagnosis should prompt a search for the underlying cause, which is not apparent at the time of angiography. The diagnosis should not be applied to patients in whom the underlying cause for the acute presentation is clini- 


\begin{tabular}{|c|c|}
\hline Clinical disorder & Diagnostic investigation \\
\hline \multicolumn{2}{|l|}{ Non-cardiac disorders } \\
\hline Renal impairment & Serum creatinine \\
\hline Pulmonary embolism & CTPA or ventilation/perfusion imaging \\
\hline \multicolumn{2}{|l|}{ Cardiac disorders } \\
\hline \multicolumn{2}{|l|}{ Myocardial disorders } \\
\hline Cardiomyopathy (takotsubo, dilated, hypertrophic) & Left ventriculography, Echo, CMR \\
\hline Myocarditis & CRP, CMR, EMB \\
\hline Myocardial trauma or injury & History (trauma, chemotherapy), CMR \\
\hline Tachyarrhythmia-induced infarct & Arrhythmia monitoring \\
\hline \multicolumn{2}{|l|}{ Coronary disorders } \\
\hline $\begin{array}{l}\text { Concealed coronary dissection (aortic dissection involving valve, } \\
\text { spontaneous coronary dissection) }\end{array}$ & Echo, CT angiogram \\
\hline Sympathomimetic-induced spasm & Drug screen (eg, cocaine) \\
\hline Epicardial coronary spasm & ACh provocation testing \\
\hline Microvascular spasm & ACh provocation testing \\
\hline Microvascular dysfunction & Coronary flow reserve \\
\hline Coronary slow-flow phenomenon & TIMI frame count \\
\hline Plaque disruption/coronary thrombus & Intravascular ultrasound \\
\hline Coronary emboli & Echo (left ventricular or valvular thrombus) \\
\hline \multicolumn{2}{|l|}{ Thrombotic disorders } \\
\hline Factor V Leiden & Thrombophilia disorder screen \\
\hline Protein C \& S deficiency & \\
\hline
\end{tabular}

$\mathrm{ACh}$, acetylcholine; CMR, cardiac magnetic resonance imaging; CRP, C-reactive protein; CT, computed tomography; CTPA, computed tomographic pulmonary angiogram; Echo, echocardiography; EMB, endomyocardial biopsy; TIMI, thrombolysis in myocardial infarction.

cally apparent at the time of angiography (as per the third diagnostic criteria listed earlier). For example, a young patient with a typical presentation of myopericarditis (eg, antecedent viral illness, fever, pleuritic chest pain, nonspecific ST changes) in whom coronary angiography was undertaken to essentially exclude CAD, should not be diagnosed with MINOCA but with 'viral myopericarditis' because no further evaluation of the underlying cause is required. In contrast, a patient with no antecedent viral illness or fever, who presents with nonspecific chest pain, ECG changes and a troponin elevation in the context of non-obstructive CAD should be diagnosed as MINOCA at the time of angiography, but may subsequently be shown to have myocarditis on CMR imaging.

\section{WHO Is at Risk of MINOCA?}

A recent systematic review of large AMI registries has detailed the typical characteristics of patients with MINOCA. In the 13 studies with available data, the median age of the MINOCA patients was 55 years and $40 \%$ were women. ${ }^{9}$ Compared with AMI patients who had obstructive CAD, those with MINOCA were more likely to be younger, female and have hyperlipidemia. ${ }^{9}$ However, delineating a MINOCA patient from one with AMI associated with obstructive CAD on the basis of clinical presentation is not feasible.

\section{WHY Diagnose MINOCA?}

Because MINOCA is a new diagnostic syndrome, the question arises as to why it should be created. There are 3 key reasons for establishing this new clinical entity, which include (1) ensuring its clinical recognition, (2) prompting the evaluation of its underlying cause, and (3) the guarded prognosis.

\section{Clinical Recognition}

Some clinicians consider coronary angiography as the gold standard test for establishing a diagnosis of AMI. ${ }^{10}$ For example, acute ST-elevation MI (STEMI) registry studies have labeled patients presenting with acute ST elevation and nonobstructive CAD on angiography as a "false-positive STEMI diagnosis". ${ }^{10,11}$ In some cases this may be appropriate if the ECG is misinterpreted. However, if the patient exhibits new ST elevation with a significant troponin rise and non-obstructive CAD on angiography, they should be diagnosed as MINOCA. Labeling such patients as "false-positive" AMI will result in these patients being dismissed as having a cardiac event and therefore discharged without further evaluation or appropriate treatment. In contrast, a diagnosis of MINOCA should flag these patients as requiring further cardiac assessment.

\section{Evaluating the Underlying Cause}

As described, a diagnosis of MINOCA should be a "working diagnosis", flagging the necessity to evaluate the patient for the potential underlying cause of this presentation. The clinical importance of this principle is exemplified by a recent case report of a patient with MINOCA who was found to have a myocardial bridge on diagnostic angiography, which was assumed to be the cause of the AMI and associated angina. ${ }^{12}$ However, comprehensive coronary hemodynamic assessment did not demonstrate an obstructive lesion associated with the myocardial bridge or evidence of distal microvascular dysfunction, but provocative spasm testing confirmed inducible 


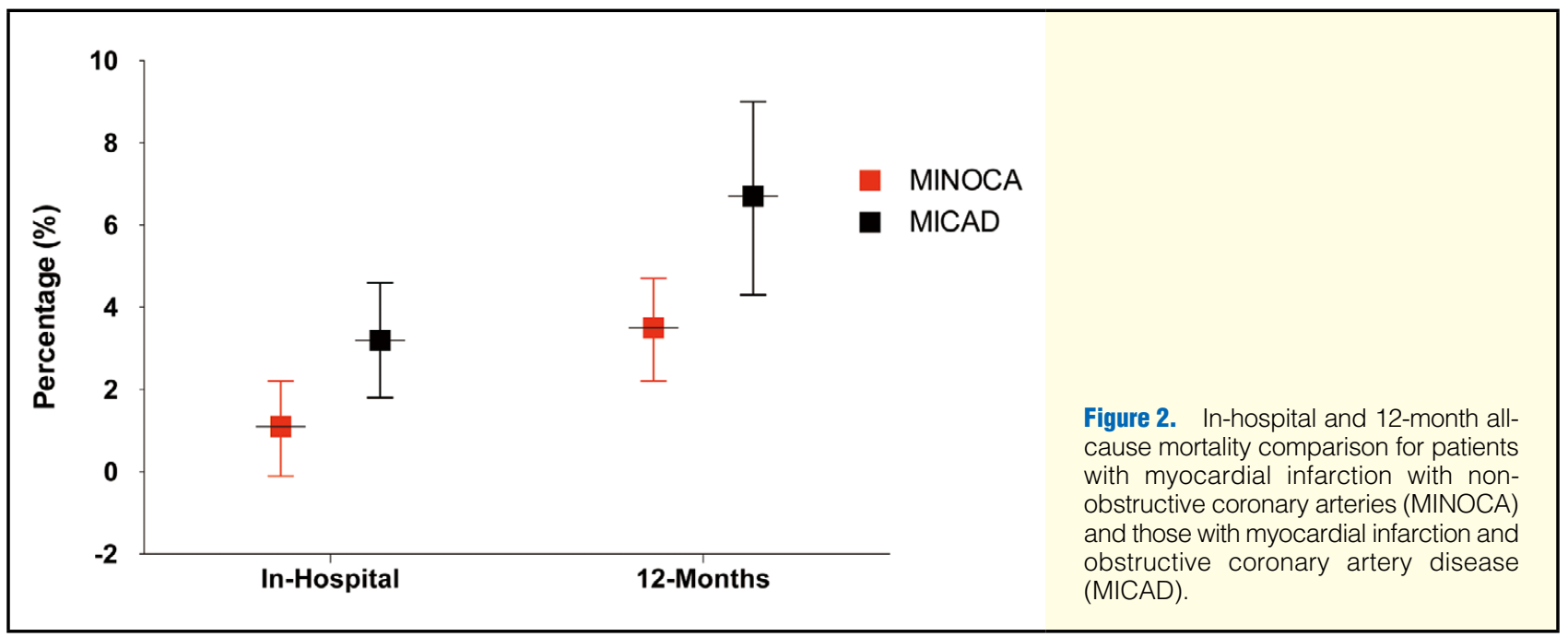

coronary spasm in the infarct-related artery. Accordingly, calcium-channel blocker therapy was instituted rather than consideration of cardiac surgery to alleviate the myocardial bridge. Moreover, studies of vasospastic angina patients have demonstrated that failure to initiate calcium-channel blocker therapy is an independent predictor of mortality. ${ }^{13}$

As exemplified by that case, identifying the underlying cause is of paramount importance in patients with MINOCA if appropriate therapy is to be instituted. The above Table summarizes the recognized causes of MINOCA. Clearly, divergent therapies are required depending upon the identified cause, thus illustrating the importance of labeling these patients as MINOCA.

\section{Guarded Prognosis}

The prognosis of patients with MINOCA is unclear and likely to be heterogeneous, considering the diverse mechanisms (Table) responsible for the syndrome. Pooled analysis of 8 studies that followed up patients with MINOCA suggests an in-hospital all-cause mortality of $0.9 \%$ (95\% confidence interval (CI): $0.5 \%, 1.3 \%)$ and $4.7 \%(95 \% \mathrm{CI}: 2.6 \%, 6.9 \%)$ at 12 months. ${ }^{9}$ Analysis of AMI registries that compared patients with an $\mathrm{AMI}$ associated with obstructive CAD with those with MINOCA suggests that the latter have a more favorable prognosis (Figure 2). However, it is disconcerting that patients with stable chest pain (who have not experienced a recent AMI) in the absence of obstructive CAD, have an annual allcause mortality of $0.3 \% .{ }^{14}$ Moreover, in the prospectively conducted Korean MI Registry, ${ }^{15}$ the MINOCA patients had the equivalent 12-month all-cause mortality to those with an AMI associated with single- or double-vessel CAD. Hence patients with MINOCA should receive the same clinical attention as AMI patients who have single- or double-vessel disease and not merely dismissed as having an insignificant clinical condition.

\section{HOW to Manage MINOCA?}

As mentioned, the key principle in the management of MINOCA is to consider it as a working diagnosis and elucidate the underlying cause for the apparent AMI. These causes may be noncardiac or cardiac (Table) and require specific evaluation and therapies.

\section{Clinical Assessment}

When the diagnosis of MINOCA is made at coronary angiography, the patient should be clinically re-evaluated in the light of the findings, with consideration given to the potential causes (Table) for the presentation. The clinical history should be re-examined, focusing on aspects such as an antecedent viral illness, prolonged immobilization, calf tenderness, recent psychological trauma, reduced exercise tolerance, drug abuse, or a family history of thrombotic disorders. The admission biochemistry should be considered and an assessment made if the elevated troponin is spurious because of impaired renal function. Other acute extracardiac conditions that may be associated with an incidental troponin rise include sepsis, stroke, respiratory distress syndrome or a sustained tachyarrhythmia, all of which should be evident from the clinical presentation.

\section{Angiographic Assessment}

Closer examination of the coronary angiogram may provide insights into the underlying cause, such as spontaneous episodes of coronary artery spasm, evidence of the coronary slow-flow phenomenon, coronary thrombi/emboli, or a subtle spontaneous coronary artery dissection. If left ventriculography is also performed, myocardial causes for MINOCA may become apparent such as takotsubo or other cardiomyopathies.

\section{Pulmonary Embolism}

Consideration of pulmonary embolism is especially important because this is a potentially fatal condition that is readily treatable. A D-dimer assay should be performed to exclude this possibility and further imaging undertaken if the results are suspicious. Routine CT pulmonary angiography for MINOCA patients is not recommended, because 1 study undertook this investigation in 100 consecutive MINOCA patients with no scans identifying a pulmonary embolus. ${ }^{16}$

\section{Myocardial Imaging}

If available, CMR imaging is particularly useful in the assessment of patients with MINOCA. This imaging technique is not only the benchmark noninvasive method of establishing a diagnosis of myocarditis (the most common abnormality detected in MINOCA patients undergoing $\mathrm{CMR}^{17,18}$ ), but is also useful in the assessment of most of the structural myocar- 
dial disorders responsible for this presentation. Furthermore, it is able to confirm the presence of a true subendocardial MI (observed in 24\% patients of MINOCA patients undergoing $\mathrm{CMR}^{9}$ ). Thus in MINOCA patients when the clinical assessment does not provide any direction to the specific cause of the presentation, CMR imaging is a useful noninvasive initial investigation. In those with a history suspicious of myocarditis or another myocardial disorder, it should also be considered early in the diagnostic pathway.

Echocardiography is more readily available than CMR and can provide useful information on myocardial function but it is not as specific as CMR in the detection of myocarditis. Moreover, poor imaging quality may restrict its utility in the diagnosis of some disorders such as takotsubo cardiomyopathy.

\section{Thrombophilia Screen}

Studies of MINOCA have reported that as many as $14 \%$ of these patients may have an abnormality detected on thrombophilia screening. ${ }^{9}$ Congenital thrombophilia disorders detected in patients with MINOCA include factor V Leiden, protein C $\&$ S deficiency, and factor XII deficiency. The pathogenetic role of these abnormalities in MINOCA is unclear and whether anticoagulation is required when they are detected is open to speculation.

\section{Substance Abuse}

Sympathomimetic agents such as cocaine ${ }^{19}$ and metamphetamines $^{20}$ may induce coronary artery spasm, thereby precipitating an AMI in the absence of obstructive CAD. Accordingly in patients with suspected substance abuse, a drug screen for these agents should be undertaken.

\section{Provocative Spasm Testing}

Vasospastic angina ${ }^{21}$ is an important potential cause of MINOCA because the use of calcium-channel blockers can prevent further cardiac events in these patients. Pooled analysis of provocative spasm testing studies in patients with MINOCA suggests that as many as $28 \%$ have inducible spasm. ${ }^{9}$ Thus some clinicians would advocate routine provocative spasm testing in patients with MINOCA. This investigation is typically undertaken in specialized centers and although considered a relatively safe investigation, it is not without risk. 22,23 The timing for undertaking provocative spasm testing following AMI has varied between studies, with some performing testing during the initial diagnostic angiogram, ${ }^{24}$ whereas others have deferred testing until at least 2 weeks later. ${ }^{25}$ Clearly, the clinical presentation of individual patients needs to be closely considered before provocative spasm is embarked upon. If provocative spasm testing is not performed early, perhaps calcium-channel blockers should be empirically prescribed until testing can be performed. Spasm provocation testing should be considered in MINOCA patients, especially if recurrent angina occurs following the initial MINOCA presentation (as illustrated in the case report ${ }^{12}$ ).

\section{Other Investigations}

Myocarditis is a common cause of MINOCA and routine investigations such as $\mathrm{C}$-reactive protein may provide clues for this diagnosis (elevated CRP) and prompt CMR imaging to be undertaken. ${ }^{18}$ Endomyocardial biopsy should be considered to confirm the presence of myocarditis, particularly in fulminant cases. ${ }^{26}$

Some researchers have demonstrated plaque disruption on intravascular ultrasound in patients with MINOCA. ${ }^{27}$ Hence this imaging modality may provide further insights into the underlying cause of MINOCA.

\section{WHERE to Next for MINOCA?}

Establishing MINOCA as a diagnostic entity has initiated a journey in improving the quality of care and our understanding of this disorder. However, determining the optimal assessment pathway in elucidating the underlying causes for this condition requires further investigation.

Future research that is warranted in MINOCA is evaluating the most appropriate treatment of these patients, particularly for those in whom no underlying pathogenetic mechanism for the AMI has been identified. Hence the MINOCA patient who has a subendocardial MI demonstrated on CMR imaging but no evidence of a coronary disorder (such as in Figure 1). Should this patient be empirically managed with aspirin and statin? Should calcium-channel blockers be initiated on the presumption of coronary spasm as the underlying mechanism or should provocative spasm testing be routinely performed prior discharge? What if there is no evidence of an AMI on CMR imaging, should these empirical therapies still be initiated? Will regular use of these preventative therapies improve the guarded prognosis of MINOCA?

Clearly there are many questions that need to be addressed in patients with MINOCA and these are best answered by multicenter collaborative studies, given the relatively low prevalence of this disorder. Hopefully, utilizing Kipling's 'six honest serving men' (ie, the "Five Ws and One H" approach) will advance the understanding and routine management of these patients as well as stimulating further research into this intriguing condition.

\section{References}

1. Hochman JS, Tamis JE, Thompson TD, Weaver WD, White HD, Van de Werf F, et al. Sex, clinical presentation, and outcome in patients with acute coronary syndromes: Global Use of Strategies to Open Occluded Coronary Arteries in Acute Coronary Syndromes IIb Investigators. N Engl J Med 1999; 341: 226-232.

2. Agewall S, Daniel M, Eurenius L, Ekenback C, Skeppholm M, Malmqvist K, et al. Risk factors for myocardial infarction with normal coronary arteries and myocarditis compared with myocardial infarction with coronary artery stenosis. Angiology 2012; 63: 500503.

3. Baccouche H, Mahrholdt H, Meinhardt G, Merher R, Voehringer M, Hill S, et al. Diagnostic synergy of non-invasive cardiovascular magnetic resonance and invasive endomyocardial biopsy in troponin-positive patients without coronary artery disease. Eur Heart $J$ 2009; 30: 2869-2879.

4. Gehani AA, al-Mulla AW, Chaikhouni A, Ammar AS, Mahrous F, Tirkawi R, et al. Myocardial infarction with normal coronary angiography compared with severe coronary artery disease without myocardial infarction: The crucial role of smoking. J Cardiovasc Risk 2001; 8: $1-8$.

5. Beltrame JF. Assessing patients with myocardial infarction and nonobstructed coronary arteries (MINOCA). J Intern Med 2013; 273: $182-185$.

6. Kipling R, Gleeson JM. Just so stories. Doubleday 1912; 83.

7. Thygesen K, Alpert JS, Jaffe AS, Simoons ML, Chaitman BR, White HD, et al. Third universal definition of myocardial infarction. Nat Rev Cardiol 2012; 9: 620-633.

8. Scanlon PJ, Faxon DP, Audet AM, Carabello B, Dehmer GJ, Eagle $\mathrm{KA}$, et al. ACC/AHA guidelines for coronary angiography: A report of the American College of Cardiology/American Heart Association Task Force on practice guidelines (Committee on Coronary Angiography): Developed in collaboration with the Society for Cardiac Angiography and Interventions. J Am Coll Cardiol 1999; 33: $1756-$ 1824.

9. Pasupathy S, Air T, Dreyer RP, Tavella R, Beltrame JF. Systematic review of patients presenting with suspected myocardial infarction and nonobstructive coronary arteries. Circulation 2015; 131: 861 870. 
10. McCabe JM, Armstrong EJ, Kulkarni A, Hoffmayer KS, Bhave PD, Garg S, et al. Prevalence and factors associated with false-positive ST-segment elevation myocardial infarction diagnoses at primary percutaneous coronary intervention-capable centers: A report from the Activate-SF registry. Arch Intern Med 2012; 172: 864-871.

11. Chung SL, Lei MH, Chen CC, Hsu YC, Yang CC. Characteristics and prognosis in patients with false-positive ST-elevation myocardial infarction in the ED. Am J Emerg Med 2013; 31: 825-829.

12. Sheikh AR, Sidharta S, Worthley MI, Yeend R, Di Fiore DP, Beltrame JF. The importance of evaluating patients with MINOCA (myocardial infarction with non-obstructive coronary arteries). Int $J$ Cardiol 2015; 199: 386-388.

13. Yasue H, Takizawa A, Nagao M, Nishida S, Horie M, Kubota J, et al. Long-term prognosis for patients with variant angina and influential factors. Circulation 1988; 78: 1 -9.

14. Di Fiore DP, Beltrame JF. Chest pain in patients with 'normal angiography': Could it be cardiac? Int J Evidence-based Healthcare 2013; 11: $56-68$.

15. Kang WY, Jeong MH, Ahn YK, Kim JH, Chae SC, Kim YJ, et al. Are patients with angiographically near-normal coronary arteries who present as acute myocardial infarction actually safe? Int J Cardiol 2011; 146: 207-212.

16. Collste O, Sorensson P, Frick M, Agewall S, Daniel M, Henareh L, et al. Myocardial infarction with normal coronary arteries is common and associated with normal findings on cardiovascular magnetic resonance imaging: Results from the Stockholm Myocardial Infarction with Normal Coronaries study. J Intern Med 2013; 273: 189 196.

17. Leurent G, Langella B, Fougerou C, Lentz PA, Larralde A, Bedossa $\mathrm{M}$, et al. Diagnostic contributions of cardiac magnetic resonance imaging in patients presenting with elevated troponin, acute chest pain syndrome and unobstructed coronary arteries. Arch Cardiovasc Dis 2011; 104: $161-170$

18. Tornvall P, Gerbaud E, Behaghel A, Chopard R, Collste O, Laraudogoitia E, et al. Myocarditis or "true" infarction by cardiac magnetic resonance in patients with a clinical diagnosis of myocardial infarction without obstructive coronary disease: A meta-analysis of individual patient data. Atherosclerosis 2015; 241: 87-91.
19. Lange RA, Hillis LD. Cardiovascular complications of cocaine use. $N$ Engl J Med 2001; 345: 351-358.

20. Hung MJ, Kuo LT, Cherng WJ. Amphetamine-related acute myocardial infarction due to coronary artery spasm. Int J Clin Pract 2003; 57: $62-64$.

21. JCS Joint Working Group. Guidelines for diagnosis and treatment of patients with vasospastic angina (coronary spastic angina) (JCS 2013): Digest version. Circ J 2014; 78: 2779-2801.

22. Takagi Y, Yasuda S, Takahashi J, Tsunoda R, Ogata Y, Seki A, et al. Clinical implications of provocation tests for coronary artery spasm: Safety, arrhythmic complications, and prognostic impact: Multicentre registry study of the Japanese Coronary Spasm Association. Eur Heart J 2013; 34: 258-267.

23. Ong P, Athanasiadis A, Borgulya G, Vokshi I, Bastiaenen R, Kubik $\mathrm{S}$, et al. Clinical usefulness, angiographic characteristics, and safety evaluation of intracoronary acetylcholine provocation testing among 921 consecutive white patients with unobstructed coronary arteries. Circulation 2014; 129: $1723-1730$.

24. Ong P, Athanasiadis A, Hill S, Vogelsberg H, Voehringer M, Sechtem U. Coronary artery spasm as a frequent cause of acute coronary syndrome: The CASPAR (Coronary Artery Spasm in Patients With Acute Coronary Syndrome) Study. J Am Coll Cardiol 2008; 52: 523-527.

25. Pristipino C, Beltrame JF, Finocchiaro ML, Hattori R, Fujita M, Mongiardo R, et al. Major racial differences in coronary constrictor response between japanese and caucasians with recent myocardial infarction. Circulation 2000; 101: 1102-1108.

26. Caforio AL, Pankuweit S, Arbustini E, Basso C, Gimeno-Blanes J, Felix SB, et al. Current state of knowledge on aetiology, diagnosis, management, and therapy of myocarditis: A position statement of the European Society of Cardiology Working Group on Myocardial and Pericardial Diseases. Eur Heart J 2013; 34: 2636-2648, 2648a2648d.

27. Reynolds HR, Srichai MB, Iqbal SN, Slater JN, Mancini GBJ, Feit $\mathrm{F}$, et al. Mechanisms of myocardial infarction in women without angiographically obstructive coronary artery disease. Circulation 2011; 124: 1414-1425. 